\title{
Prevalence of Rheumatic Heart Disease in Children and Young
}

\section{Adults in Nicaragua}

\author{
John A. Paar, MD ${ }^{\mathrm{a}}$, Nubia M. Berrios, $\mathrm{MD}^{\mathrm{b}}$, John D. Rose, $\mathrm{MD}^{\mathrm{C}}$, Mercedes Cáceres, MD, \\ PhD $^{d}$, Rodolfo Peña, MD, PhD ${ }^{e}$, Wilton Pérez, MS $^{e}$, Mario Chen-Mok, PhD $^{f}$, Erik Jolles, BA $^{f}$, \\ and James B. Dale, MDg \\ aWakeMed Hospital, Raleigh, NC \\ bDepartment of Pediatrics, National Autonomous University of Nicaragua at León \\ 'East Carolina University School of Medicine, Greenville, NC

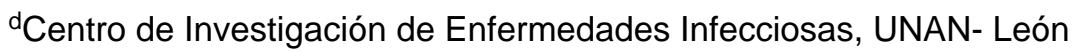 \\ eCentro de Investigación en Demografía y Salud, UNAN-León \\ ${ }^{f} F$ amily Health International, Durham, NC \\ gUniversity of Tennessee Health Science Center and the Veterans Affairs Medical Center, Memphis, \\ TN
}

\begin{abstract}
Rheumatic heart disease (RHD) results in morbidity and mortality that is disproportionate among people in developing countries compared to those living in economically developed countries. The global burden of disease is uncertain because most previous studies to determine the prevalence of RHD in children relied on clinical screening criteria that lacked the sensitivity to detect most cases. The present study was performed to determine the prevalence of RHD in children and young adults in León, Nicaragua, an area previously thought to have a high prevalence of RHD. This was an observational study of 3150 children, ages 5-15, and 489 adults, ages 20-35, randomly selected from urban and rural areas of León. Cardiopulmonary exams and echo-Doppler studies were performed on all subjects. Echo-Doppler diagnosis of RHD was based on pre-defined consensus criteria that were developed by a WHO/NIH working group. The overall prevalence of RHD in children was $48 / 1000$ (95\% C.I. $=35 / 1000-60 / 1000$. The prevalence in urban children was $34 / 1000$ and in rural children it was $80 / 1000$. Using more stringent echo-Doppler criteria designed to diagnose definite RHD in adults, the prevalence was 22/1000 (95\% C.I. $=8 / 1000-37 / 1000)$. In conclusion, the prevalence of RHD among children and adults in this economically disadvantaged population far exceeds previously predicted rates. The findings underscore the potential health and economic burden of acute rheumatic fever and RHD and support the need for more effective measures of prevention, which may include safe, effective and affordable vaccines to prevent the streptococcal infections that trigger the disease.
\end{abstract}

Corresponding Author: James B. Dale, MD, University of Tennessee Health Science Center, 20 S. Dudley St., Suite 804, Memphis, TN 38163, jbdale@uthsc.edu, ph: (901) 448-2101, fax: (901) 448-8231.

Publisher's Disclaimer: This is a PDF file of an unedited manuscript that has been accepted for publication. As a service to our customers we are providing this early version of the manuscript. The manuscript will undergo copyediting, typesetting, and review of the resulting proof before it is published in its final citable form. Please note that during the production process errors may be discovered which could affect the content, and all legal disclaimers that apply to the journal pertain. 


\section{Keywords}

rheumatic heart disease; prevalence of disease; disease burden

\section{Introduction}

Prior to the availability of echo-Doppler technology, the diagnosis of RHD was made on the basis of the clinical history and physical findings. Two previous reports 1,2 have presented evidence that the clinical examination lacks sensitivity in detecting RHD in high-risk populations. Marijon, et al, found that echocardiography detected ten times the number of cases of RHD compared to clinical exam alone in large studies of children in Cambodia and Mozambique. $^{2}$ The present study was undertaken based upon extensive experience by one of us (J.A.P.) conducting a cardiology clinic in León, Nicaragua over the past 24 years indicating that the prevalence of RHD was probably high and that the current approach of only identifying and treating patients with known RHD based on clinical findings was inadequate to address the magnitude of the problem. Inasmuch as there are current efforts to produce an effective vaccine against GAS, which could potentially prevent ARF, we wished to establish the true prevalence of RHD in the population using both the physical examination and echo-Doppler criteria.

\section{Methods}

We performed an observational community-based study during 2006-2009 of 3150 children living in the municipality of León, Nicaragua. Dwellings were randomly selected from the population data base of the demographic surveillance system of the Centro de Investigación en Demografía Salud (CIDS) of the National Autonomous University of Nicaragua at León (UNAN-León). The CIDS surveillance population corresponds to clusters selected with probability proportional to size from the entire population of León. ${ }^{3}$ After approval by the Minister of Health in Nicaragua, the Ethics Committees of UNAN and the University of Tennessee Health Science Center, all children in selected dwellings between the ages of 5 and 15 years were invited to participate in the study. Consent was obtained from parents and from children 13 years or older. All children whose parents gave permission to UNAN-CIDS surveyors were transported to the pediatric cardiology clinic of the Hospital Escuela Oscar Danilo Rosales Argüello (HEODRA). A clinical report form was completed by a study nurse after interviewing the parent and subject. Examination of the lungs and heart was performed by a cardiologist and an opinion was rendered as to the presence or absence of clinical evidence of rheumatic heart disease. An echo-Doppler study was performed on all subjects using a General Electric Vivid i digital machine. Studies were performed by a pediatric cardiologist (N.B.) or by one of four qualified visiting echocardiographic technicians. Echocardiograms included parasternal long and short axis and apical 3 and 4 chamber views. Normal subjects and those with evidence of congenital heart disease without RHD exited the study. EchoDoppler studies with abnormalities consistent with RHD were stratified as definite, probable, or possible RHD, as defined by an expert panel convened under the auspices of the W.H.O. and the National Institutes of Health in September, 2005 (Table 1).

All subjects classified as "probable" or "possible" RHD were scheduled for repeat clinical and echocardiographic evaluation within 4-12 months (target 6 months) following the initial encounter. All subjects meeting any of the diagnostic criteria for RHD were referred to the RHD clinic at HEODRA to receive antibiotic prophylaxis and follow-up care which was not part of the investigative protocol. Subjects were then reclassified based upon the results of the second examination. The data were analyzed according to health center region, urban vs. rural, age and sex, and changes in diagnostic status with subsequent studies. All echo-Doppler studies 
that showed abnormalities consistent with definite, probable, or possible RHD were read independently by two cardiologists and any discrepancies in reading were resolved either by review of the study and agreement between the readers or by submission to a third cardiologist as a tie-breaker. All follow-up studies were read by 2 cardiologists and in all cases the echocardiograms were recorded in crude Dicom. Abnormal studies and follow-up studies were recorded as MPEGs and retained for subsequent review.

Because RHD in some subjects may manifest as a progressive chronic disease of the cardiac valves, we subsequently performed an additional study to assess the prevalence of RHD in young adults between the ages of 20 and 35 . Selection of adult participants followed procedures similar to those used to select the pediatric participants. Because it was likely that cardiac pathology unrelated to RHD would be more prevalent in this age group, only two classifications of RHD were specified, with the intent of excluding non-rheumatic etiologies by requiring anatomic changes typical of RHD and not Doppler abnormalities alone (Table 2). Cardiopulmonary examinations and echo-Doppler studies were performed on all subjects as previously described above. Echo-Doppler studies with abnormalities consistent with RHD were reviewed independently by two cardiologists and the results were categorized into Group 1 or Group 2 as described (Table 2).

The sample size for the study in children was calculated based on an anticipated prevalence of RHD of 3/1000 (WHO Technical Report series 923) to estimate the prevalence of RHD within 0.2 percent points with a $95 \%$ confidence interval. These calculations also assumed a 1.2 design effect due clustering in the sampling design. Assuming a 10\% non-response rate, we targeted a total of 3600 children. Because the non-response rate plus migrations and missed appointments was approximately $20 \%$, an additional random sample of 1000 children was drawn to complement the initial sample. At the time the adult study was designed, we calculated a rate of RHD of 33/1000 in children. We determined that a sample size of 500 would allow us to obtain an estimate for adults with a $95 \%$ confidence bounds of plus or minus 0.016 $(16 / 1000)$.

We estimated the prevalence per 1000 subjects of the different RHD diagnosis categories and by gender and location (rural/urban). We computed $95 \%$ confidence intervals for prevalence estimates and tested the differences between some of these groups using design-adjusted chisquare tests. The Taylor series expansion method to estimate standard errors, as implemented by SAS 9.2, was used to account for clustering effects as appropriate for the sampling design.

\section{Results}

Of the 3150 children that participated in the study, the mean age was 9.5 years and the median age was $10.7,51 \%$ were male and $49 \%$ female, $70 \%$ were from an urban neighborhood and $30 \%$ were classified as rural (Table 3). Results from the initial examination revealed an overall prevalence of RHD of 48/1000 (95\% C.I. $=35 / 1000-60 / 1000)$ with the majority of subjects meeting the criteria for "possible" RHD (Table 4). Fifty five percent of subjects with RHD were female. There was a significantly higher prevalence of RHD in subjects from rural compared to urban environments ( $77 / 1000$ vs. $35 / 1000, \mathrm{p}<0.0001)$ (Table 5). Of the 42 children with congenital heart disease ( $1.3 \%$ of the total), the vast majority had congenitally abnormal aortic valves.

Follow-up examinations were performed 4-12 months after the initial exam (median= 5.7 months) on subjects that met the diagnostic criteria for "possible" or "probable" RHD in order to assess changes in the clinical and echo-Doppler findings over time (Table 6). Repeat echoDoppler studies on the majority of the subjects $(73 / 126,58 \%)$ that were initially categorized as "possible" RHD exhibited little or no change. Eleven subjects (9\%) with "possible" RHD 
were lost to follow-up. Eleven of the 126 subjects (9\%) developed a murmur of mitral regurgitation (MR) associated with anatomic abnormalities of the mitral valve that resulted in re-classifying them in the "definite" RHD category. Of those who remained classified as "possible" RHD, review of the studies showed progression of anatomical changes or worsening MR in 18 subjects (14\%). These changes included development of or progression in mitral leaflet thickening, the development of the so-called "dog-leg" or "elbow" deformity of the mitral valve, further restriction in leaflet motion, and an increase in the length and width of the mitral regurgitant jet. However, it was also observed that 41/126 subjects with "possible" RHD on the first examination no longer met the diagnostic criteria and were reclassified as "normal" on the second examination. It was noted that this was in fact a very mixed group. Four subjects initially found to have significant jets of aortic regurgitation (AR) and aortic valve thickening reverted to completely normal. One child with a murmur of MR on the first examination and a $3.2 \mathrm{~cm}$ jet of MR by echocardiogram was subsequently found to have thickness of the mitral and aortic leaflets but no regurgitant jet of either valve. Others had deformity or thickness of the MV leaflets and often had posterolaterally directed jets of MR, but no longer met the $2 \mathrm{~cm}$ minimum regurgitant jet criterion and thus were reclassified as "normal". In all, only 20 of the group initially called "possible" RHD showed no anatomic or Doppler abnormality on the second examination, of whom 4 had had AR and the remainder had MR on the first examination.

Re-examination of the 8 subjects that initially met the criteria for "probable" RHD yielded variable results as well. Four subjects no longer had an audible murmur and reverted to "possible" RHD, 2 subjects showed no anatomic change and no murmur, 1 had anatomic changes of the mitral valve and was reclassified as "definite" RHD, and 1 remained in the "probable" RHD category.

The variable results of follow-up examinations on pediatric subjects in the "possible" and "probable" RHD categories prompted us to extend these studies to include a group of young adult residents of León. We reasoned that because the natural history of RHD in children may vary depending on the severity of the initial ARF carditis, the valves affected, the number of recurrent attacks of ARF and age of onset 4, a sub-study of young adults could provide some indication of the prevalence of RHD in the age group more likely to be affected by the chronic progressive form of the disease. ${ }^{5}$ The mean age of the 489 adult subjects enrolled was 26.8 , which included 184 males (38\%) and 305 females (62\%). 316 (65\%) were from urban neighborhoods and $173(35 \%)$ were from rural areas (Table 3 ). The predominance of female participants was apparently due to the absence of males from the selected dwellings when the interviewers appeared together with a much higher percentage of males that refused to participate. The echo-Doppler criteria for RHD were more stringent than in the pediatric study and included only those with definite structural abnormalities of the mitral valve. Isolated aortic valve lesions in the absence of mitral valve abnormalities were excluded because of the potential for overlap with non-rheumatic aortic valve disease in this age group. Of the 489 subjects enrolled, the prevalence of "definite" RHD with or without physical findings was $22 / 1000$ (95\% C.I. $=8 / 1000-37 / 1000), 16 / 1000$ without physical findings and 6/1000 with physical findings (Table 7). The prevalence of definite RHD in urban residents was 25/1000 vs. $17 / 1000$ ( $\mathrm{p}=0.9071$ ) in rural residents (Table 7). None of the subjects provided a history of cardiovascular symptoms. None had a history of ARF or symptoms consistent with ARF or a previous examination showing evidence of RHD.

\section{Discussion}

The results of the present study confirm that the prevalence of RHD in school-aged children is far greater than earlier estimates and extend the observations to show a high prevalence of disease in young adults who may be at greater risk of developing chronic progressive RHD. ${ }^{6}$ 
The use of echocardiography to diagnose RHD is now introducing additional challenges in interpretation of the results. Previous authors have warned of the potential of over diagnosis, particularly in the setting of ARF, by using such a highly sensitive technology. ${ }^{7-} 9$ The echoDoppler criteria for "possible", "probable", and "definite" RHD used in this study were adopted to reflect anatomic and hemodynamic abnormalities associated with RHD that would lie outside the realm of normal physiologic variants. The color Doppler criteria, including the jet lengths and characteristics, clearly exceed those seen in normal children.10,11 Minich, et al. 12 have suggested that the posterolateral orientation and the length of the regurgitant jet, presence in two planes, high velocity (mosaic appearance), and holosystolic duration, make the positive predictive value of RHD MR virtually $100 \%$.

We believe the echo-Doppler criteria used in this study are sufficiently stringent to determine prevalence rates of RHD in a population at high risk for the disease. However, as additional similar studies are reported, it will be necessary to determine the relationship of the echoDoppler data to the natural history of the disease and eventual clinical outcomes. Because it is neither ethical nor desirable to diagnose RHD in childhood and not provide secondary antibiotic prophylaxis to prevent recurrent attacks of ARF, it would be impossible to perform a longitudinal study to correlate echo-Doppler findings with eventual clinical outcome in a cohort of untreated subjects. Therefore, we opted to perform short-term follow-up studies on children with "possible" and "probable" RHD and also to study a group of young adults from the same population in order to extrapolate the progression of disease over time. In children with "possible" RHD on the initial exam, repeat evaluations of 126 cases showed that $33 \%$ reverted to normal or showed improvement such that they no longer met the criteria for RHD, 58\% remained unchanged and $9 \%$ worsened and were re-categorized as "probable" or "definite" RHD. It is possible that antibiotic prophylaxis had some impact on these results by preventing intercurrent attacks of ARF. However, given the short duration of follow-up and the number of subjects that improved it is unlikely that antibiotic treatment had a significant effect on the outcome. Alternatively, our results are consistent with previous limited longitudinal studies of 66 ARF patients with "mild" carditis. 13 These authors reported that $39 \%$ remained clinically unchanged, $5 \%$ progressed and $56 \%$ were clinically normal (no murmur). Fifteen patients in the latter group were evaluated by follow-up echocardiograms and 7 (47\%) had normal exams. 13 The results from our cohort of young adults showed a prevalence of "definite" RHD of $22 / 1000$, suggesting that in this population approximately half of the children with echoDoppler abnormalities may develop chronic RHD later in life. Although females outnumbered males (62\% vs. $38 \%$ ) in our adult study, this does not completely explain the finding that $91 \%$ of cases of definite RHD were females. Although there is a slight predominance of female children with RHD in this study (55\%) and in others, 14,15 this ratio of adult females with RHD was unexpected.

We believe the most important reason to establish a valid assessment of RHD prevalence is to provide information about the disease burden so that public health officials may make informed decisions regarding the appropriation of scarce resources to reduce disease incidence based on cost-effective measures. Others have supported the idea of using echocardiography as a universal screening procedure to identify children at risk of recurrent attacks of ARF that may benefit from secondary prophylaxis with penicillin. ${ }^{2}$ Although the efficacy of secondary prevention has been established for decades, ${ }^{16}$ our experience in Nicaragua indicated that compliance with prophylaxis regimens was very low and, as others have indicated, could potentially only be improved by implementing a costly registry system manned by public health officials. ${ }^{17} \mathrm{We}$ conclude that establishing a more realistic prevalence of RHD will add impetus to efforts aimed at the clinical development and deployment of safe, effective and affordable vaccines to prevent the GAS infections that cause ARF and RHD. ${ }^{18}$ 
Results of the current study and those from others $1,2,19$ provide information that may be used to calculate new estimates of the burden of RHD in developing countries. Echocardiographic diagnostic criteria resulted in prevalence rates of 62/1000 in Kenya, 121.5/1000 in Cambodia, 30.4/1000 in Mozambique ${ }^{2}$ and 48/1000 in Nicaragua (this report), providing an average point prevalence of 40/1000 in school age children. A previous prospective evaluation of children with ARF in the modern era in Brazil indicated that $72 \%$ of the 258 subjects developed chronic valvular disease and $16 \%$ progressed to severe aortic and/or mitral disease. ${ }^{5}$ Thus, previous reports of the global burden of RHD20 may be underestimated by a factor of 4-5. Based on this assumption, the new estimates would suggest that 62-78 million individuals worldwide may currently have RHD that could potentially result in 1.4 million deaths/yr from RHD and its complications. There is now sufficient evidence to indicate that clinical criteria alone underestimate RHD prevalence by 5-10 fold compared to echocardiographic criteria. We believe it is reasonable to assume that the actual prevalence and predicted disease burden lies somewhere between these two estimates and should provide greater impetus for local and global strategies for prevention.

\section{Acknowledgments}

This study was supported by research funds from USPHS grant 5U01AI060592 and was performed in León, Nicaragua.

This study was supported by research funds from USPHS grant 5U01AI060592. Dr. Fran Rubin and Ms. Robin Mason (NIH/NIAID) provided significant input into the design and implementation of the protocol as well as outstanding assistance for logistics and coordination of personnel. We especially thank echocardiographic technicians Wendy Barnhardt, Chris Baumann, and Ruben Centeno. We appreciate the participation of Bruce Alpert, MD, Professor of Pediatric Cardiology, the University of Tennessee Health Science Center, Memphis, TN and Cindy Rosson, echocardiographic technician. We also thank the following personnel of CIDS, Centro Para la Investigación Demográfica de Salud: Marisol Benevides, quality control; Maria Lourdes Martínez, in charge of the data base; Noelia Jaen, data base; Janeth Miranda and Sandra Esquivel, clinic nurses; and Lidia M. Rojas Ch, Hilda V. Amaya, and Eduardo Toval, surveyors. Most importantly, we thank the children, their parents, and the adults from León, Nicaragua who freely participated in the study.

\section{References}

1. Anabwani GM, Bonhoeffer P. Prevalence of heart disease in school children in rural Kenya using colour-flow echocardiography. East Afr Med J 1996;73:215-217. [PubMed: 8706601]

2. Marijon E, Ou P, Celermajer DS, Ferreira B, Mocumbi AO, Jani D, Paquet C, Jacob S, Sidi D, Jouven $\mathrm{X}$. Prevalence of rheumatic heart disease detected by echocardiographic screening. N Engl J Med 2007;357:470-476. [PubMed: 17671255]

3. Pena R, Perez W, Melendez M, Kallestal C, Persson LA. The Nicaraguan Health and Demographic Surveillance Site, HDSS-Leon: a platform for public health research. Scand J Public Health 2008;36:318-325. [PubMed: 18519303]

4. Bland EF, Jones TD. The natural history of rheumatic fever: a 20 year perspective. Ann Intern Med 1952;37:1006-1026. [PubMed: 12986608]

5. Meira ZM, Goulart EM, Colosimo EA, Mota CC. Long term follow up of rheumatic fever and predictors of severe rheumatic valvar disease in Brazilian children and adolescents. Heart 2005;91:1019-1022. [PubMed: 16020588]

6. Carapetis JR, McDonald M, Wilson NJ. Acute rheumatic fever. Lancet 2005;366:155-168. [PubMed: 16005340]

7. Narula J, Chandrasekhar Y, Rahimtoola S. Diagnosis of active rheumatic carditis. The echoes of change. Circulation 1999;100:1576-1581. [PubMed: 10510063]

8. Narula J, Kaplan EL. Echocardiographic diagnosis of rheumatic fever. Lancet 2001;358:2000. [PubMed: 11747959]

9. Vasan RS, Shrivastava S, Vijayakumar M, Narang R, Lister BC, Narula J. Echocardiographic evaluation of patients with acute rheumatic fever and rheumatic carditis. Circulation 1996;94:73-82. [PubMed: 8964121] 
10. Brand A, Dollberg S, Keren A. The prevalence of valvular regurgitation in children with structurally normal hearts: a color Doppler echocardiographic study. Am Heart J 1992;123:177-180. [PubMed: 1729823]

11. Minich, L.; Tani, L.; Veasey, L. American Registry of Pathology, Armed Forces Institute of Pathology. Washington D.C.: 1999. Role of echocardiography in the diagnosis and follow-up evaluation of rheumatic carditis. ISBN1-881041-47-6

12. Minich LL, Tani LY, Pagotto LT, Shaddy RE, Veasy LG. Doppler echocardiography distinguishes between physiologic and pathologic "silent" mitral regurgitation in patients with rheumatic fever. Clin Cardiol 1997;20:924-926. [PubMed: 9383585]

13. Veasey LG, Tani LY, Hill HR. Persistence of acute rheumatic fever in the intermountain area of the United States. J Pediatr 1994;124:9-16. [PubMed: 7802743]

14. Marijon E, Celermajer DS, Tafflet M, El-Haou S, Jani DN, Ferreira B, Mocumbi AO, Paquet C, Sidi $\mathrm{D}$, Jouven X. Rheumatic heart disease screening by echocardiography: the inadequacy of world health organization criteria for optimizing the diagnosis of subclinical disease. Circulation 2009;120:663668. [PubMed: 19667239]

15. Steer AC, Kado J, Wilson N, Tuiketei T, Batzloff M, Waqatakirewa L, Mulholland EK, Carapetis JR. High prevalence of rheumatic heart disease by clinical and echocardiographic screening among children in Fiji. J Heart Valve Dis 2009;18:327-335. [PubMed: 19557993]

16. Stollerman GH, Rusoff JH. Prophylaxis against group A streptococcal infections in rheumatic fever patients; use of new repository penicillin preparation. J Am Med Assoc 1952;150:1571-1575. [PubMed: 12990472]

17. Carapetis JR, Mayosi BM, Kaplan EL. Controlling rheumatic heart disease in developing countries. Cardiovasc J S Afr 2006;17:164-165. [PubMed: 17001417]

18. Dale JB. Current status of group A streptococcal vaccine development. Adv Exp Med Biol 2008;609:53-63. [PubMed: 18193657]

19. Carapetis JR. Rheumatic heart disease in Asia. Circulation 2008;118:2748-2753. [PubMed: 19106399]

20. Carapetis JR, Steer AC, Mulholland EK, Weber M. The global burden of group A streptococcal diseases. Lancet Infect Dis 2005;5:685-694. [PubMed: 16253886] 


\section{Table 1}

\section{Diagnostic criteria for rheumatic heart disease in children}

\section{Definite Rheumatic Heart Disease (either A or B):}

A. Significant mitral stenosis, defined as echocardiographic evidence of mitral stenosis with a mean diastolic pressure gradient across the mitral valve of greater than $4 \mathrm{~mm}$ and clinical findings of mitral stenosis with or without other valvular lesions. Such abnormalities as dog-leg deformity of the anterior mitral valve leaflet, fixed or restricted mitral leaflet abnormality, calcification, and commissural thickening were expected

B. The presence of a heart murmur consistent with any combination of mitral regurgitation or aortic regurgitation and echo-Doppler evidence of rheumatic valvular damage, defined as any of:

(1) significant mitral regurgitation, with a mitral regurgitation jet of at least $2 \mathrm{~cm}$ from the coaptation point of the valve leaflets, seen in 2 planes, high velocity (mosaic pattern), holosystolic, plus thickened mitral valve leaflets and/or dog-leg deformity of the anterior mitral leaflet. Additional changes might include multiple regurgitant jets, especially posterolaterally directed.

(2) Significant aortic regurgitation, defined as an aortic regurgitation jet at least $1 \mathrm{~cm}$ from the coaptation point of the valve leaflets, of high velocity, seen in 2 planes, plus thickened mitral leaflets and/or dog-leg deformity, without another apparent etiology for the aortic insufficiency, such as a bicuspid aortic valve or annuloaortic ectasia. Aortic stenosis might be associated, but aortic stenosis without associated rheumatic mitral valve disease was not accepted as evidence of rheumatic valvular disease.

\section{Probable Rheumatic Heart Disease}

The presence of a heart murmur consistent with any combination of mitral regurgitation or aortic regurgitation, and the subject comes from a population with known or suspected high rates of acute rheumatic fever and/or rheumatic heart disease and no past history of definite or probable acute rheumatic fever, and any of the following findings are present on echocardiography:

(1) Thickened mitral valve leaflets and/or dog-leg deformity of the anterior mitral valve leaflet without significant mitral stenosis.

(2) Significant mitral regurgitation, as defined under Definite rheumatic heart disease, without thickened mitral valve leaflets and/or dog-leg deformity of the anterior mitral valve leaflet.

(3) Significant aortic regurgitation, as defined under Definite rheumatic heart disease, without thickened mitral valve leaflets and/or dog-leg deformity of the anterior mitral valve leaflet.

\section{Possible Rheumatic Heart Disease}

The absence of a valvular heart murmur in a subject from a population with known or suspected high rates of acute rheumatic fever and/or rheumatic heart disease with any of the following echo-Doppler changes:

(1) Thickened mitral valve leaflets and/or dog-leg deformity of the anterior mitral valve leaflet.

(2) Significant mitral regurgitation, as defined under Definite rheumatic heart disease.

(3) Significant aortic regurgitation, as defined under Definite rheumatic heart disease. 


\section{Table 2}

Diagnostic criteria for rheumatic heart disease in adults

Definite Rheumatic Heart Disease with Physical Findings

Structural abnormalities of the mitral valve compatible with rheumatic heart disease and the presence of a heart murmur thought to be of valvular origin. The criteria were otherwise the same as for the pediatric study (see Table 1). Aortic valve thickening with aortic regurgitation, in the absence of rheumatic changes of the mitral valve, were not considered diagnostic of rheumatic heart disease.

Definite Rheumatic Heart Disease without Physical Findings

Echo-Doppler evidence of anatomic changes of the mitral valve compatible with rheumatic heart disease (see Table 1) in the absence of a murmur thought to be of valvular origin. Aortic valve thickening with aortic regurgitation were not accepted diagnostic criteria in the absence of rheumatic changes of the mitral valve. 


\section{Table 3}

Characteristics of subjects

\begin{tabular}{lcc}
\hline Age (years) & $\begin{array}{c}\text { Pediatric subjects } \\
(\mathbf{N = 3 1 5 0 )}\end{array}$ & $\begin{array}{c}\text { Adult subjects } \\
(\mathbf{N = 4 8 9 )}\end{array}$ \\
\hline Mean & 9.5 & 27.4 \\
Median & 10.7 & 26.8 \\
Range & $5-15$ & $20-35.9$ \\
Male & $1619(51 \%)$ & $184(38 \%)$ \\
Female & $1531(49 \%)$ & $305(62 \%)$ \\
Urban & $2206(70 \%)$ & $316(65 \%)$ \\
Rural & $944(30 \%)$ & $173(35 \%)$ \\
\hline
\end{tabular}

Am J Cardiol. Author manuscript; available in PMC 2011 June 15. 


\section{Table 4}

Results of the initial cardiovascular evaluation in pediatric study participants

\begin{tabular}{lccc}
\hline $\begin{array}{l}\text { Rheumatic heart disease } \\
\text { diagnostic category }\end{array}$ & Number & Male/Female & Prevalence \\
\hline Normal & 2959 & $1522 / 1437$ & N/A \\
Possible & 137 & $59 / 78$ & $43 / 1000$ \\
Probable & 8 & $6 / 2$ & $3 / 1000$ \\
Definite & 5 & $2 / 3$ & $2 / 1000$ \\
Congenital heart disease ${ }^{*}$ & 42 & $30 / 12$ & $13 / 1000$ \\
Total & 150 & $67 / 83$ & $48 / 1000$ \\
\hline
\end{tabular}

* One participant met the diagnostic criteria for congenital heart disease and definite rheumatic heart disease 


\section{Table 5}

Urban vs. rural residence of pediatric subjects with rheumatic heart disease

\begin{tabular}{lcc}
\hline Rheumatic heart disease diagnostic category & Urban & Rural \\
\hline Possible & 67 & 70 \\
Probable & 6 & 2 \\
Definite & 4 & 1 \\
Prevalence of rheumatic heart disease & $35 / 1000$ & $77 / 1000$ \\
\hline
\end{tabular}


$\frac{0}{0}$
$\frac{0}{10}$

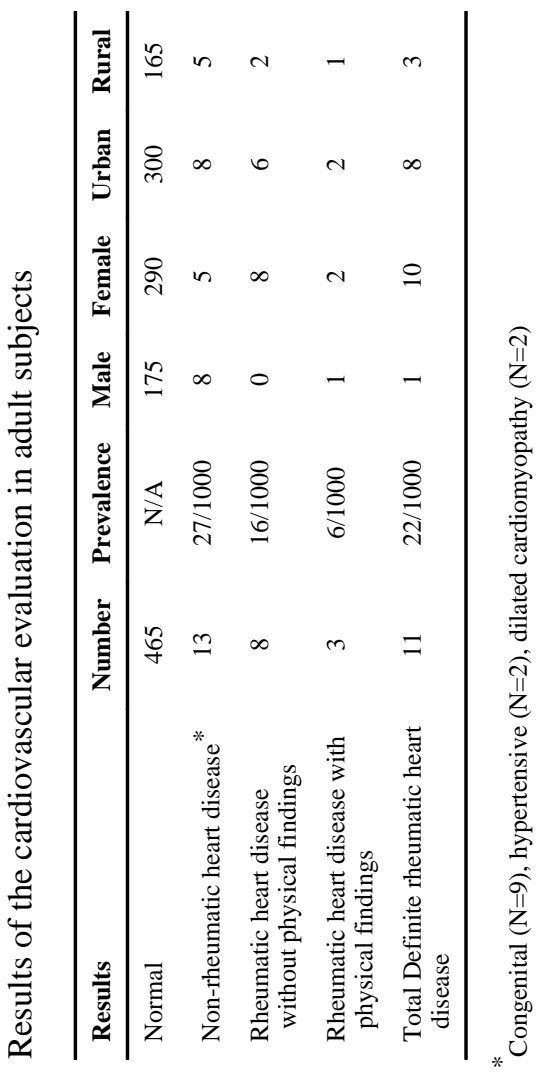

Am J Cardiol. Author manuscript; available in PMC 2011 June 15. 